\title{
Foreword: A Very Short Guide to Nudging
}

\author{
Cass R. Sunstein
}

Some policies take the form of mandates and bans. For example, the criminal law forbids theft and assault. Other policies take the form of economic incentives (including disincentives), such as subsidies for renewable fuels, fees for engaging in certain activities, or taxes on gasoline and tobacco products. Still other policies take the form of nudges - liberty-preserving approaches that steer people in particular directions but that also allow them to go their own way. In recent years, both private and public institutions have shown mounting interest in the use of nudges, because they generally cost little and have the potential to promote economic and other goals (including public health).

In daily life, a GPS is an example of a nudge; so is an "app" that tells people how many calories they ate during the previous day; so is a text message, informing customers that a bill is due or that a doctor's appointment is scheduled for the next day; so is an alarm clock; so is automatic enrollment in a pension plan; so are the default settings on computers and cell phones; so is a system for automatic payment of credit-card bills and mortgages. In government, nudges include graphic warnings for cigarettes; labels for energy efficiency or fuel economy; "nutrition facts" panels on food; the "Food Plate," which provides a simple guide for healthy eating (see choosemy plate.gov); default rules for public-assistance programs (as in "direct certification" of the eligibility of poor children for free school meals); 
a website like data.gov or data.gov.uk, which makes a large number of data sets available to the public; and even the design of government websites, which list certain items first and in large fonts.

\section{NUDGES MAINTAIN FREEDOM OF CHOICE}

It is important to see that the goal of many nudges is to make life simpler, safer, or easier for people to navigate. Consider road signs, speed bumps, disclosure of health-related or finance-related information, educational campaigns, paperwork reduction, and public warnings. When officials reduce or eliminate paperwork requirements, and when they promote simplicity and transparency, they are reducing people's burdens. Some products (such as cell phones and tablets) are intuitive and straightforward to use. Similarly, many nudges are intended to ensure that people do not struggle when they seek to interact with government or to achieve their goals.

It is true that some nudges are properly described as a form of "soft paternalism," because they steer people in a certain direction. But even when this is so, nudges are specifically designed to preserve full freedom of choice.

\section{TRANSPARENCY AND EFFECTIVENESS}

Any official nudging should be transparent and open rather than hidden and covert. Indeed, transparency should be built into the basic practice. Suppose that a government (or a private employer) adopts a program that automatically enrolls people in a pension program, or suppose that a large institution (say, a chain of private stores, or those who run cafeterias in government buildings) decides to make healthy foods more visible and accessible. In either case, the relevant action should not be hidden in any way. Government decisions in particular should be subject to public scrutiny and review. Nudges should never take the form of manipulation or trickery. The public should be able to review and scrutinize nudges no less than government actions of any other kind. 
All over the world, nations have become keenly interested in nudges. The growing interest in nudges stems from the fact that they usually impose low (or no) costs, because they sometimes deliver prompt results (including significant economic savings), because they maintain freedom, and because they can be highly effective. In some cases, nudges have a larger impact than more expensive and more coercive tools. For example, default rules, simplification, and uses of social norms have sometimes been found to have even larger impacts than significant economic incentives.

In the context of retirement planning, automatic enrollment has proved exceedingly effective in promoting and increasing savings. In the context of consumer behavior, disclosure requirements and default rules have protected consumers against serious economic harm, saving many millions of dollars. Simplification of financialaid forms can have the same beneficial effect in increasing college attendance by making it easier to access thousands of dollars in additional aid (per student). Informing people about their electricity use, and how it compares to that of their neighbors, can produce the same increases in conservation as a significant spike in the cost of electricity. If properly devised, disclosure of information can save both money and lives. Openness in government, disclosing both data and performance, can combat inefficiency and even corruption.

\section{THE NEED FOR EVIDENCE AND TESTING}

For all policies, including nudges, it is exceedingly important to rely on evidence rather than intuitions, anecdotes, wishful thinking, or dogmas. The most effective nudges tend to draw on the most valuable work in behavioral science (including behavioral economics), and hence reflect a realistic understanding of how people will respond to government initiatives. Some policies, including some nudges, seem promising in the abstract but turn out to fail in practice. Empirical tests, including randomized controlled trials, are indispensable. Bad surprises certainly are possible, including unintended adverse consequences, and sensible policymakers must try to anticipate such surprises in advance (and to fix them if they 
arise). Sometimes empirical tests reveal that the planned reform will indeed work - but that some variation on it, or some alternative, will work even better.

Experimentation, with careful controls, is a primary goal of the nudge enterprise. Fortunately, many nudge-type experiments can be run rapidly and at low cost, and in a fashion that allows for continuous measurement and improvement. The reason is that such experiments sometimes involve small changes to existing programs, and those changes can be incorporated into current initiatives with relatively little expense or effort. If, for example, officials currently send out a letter to encourage people to pay delinquent taxes, they might send out variations on the current letter and test whether the variations are more effective.

\section{TEN IMPORTANT NUDGES}

Nudges span an exceedingly wide range, and their number and variety are constantly growing. Here is a catalogue of ten important nudges:

1. Default rules (e.g., automatic enrollment in programs, including education, health, savings)

2. Simplification (in part to promote take-up of existing programs)

3. Uses of social norms (emphasizing what most people do, e.g., "Most people plan to vote," or "Most people pay their taxes on time," or "Nine out of ten hotel guests reuse their towels.")

4. Increases in ease and convenience (e.g., making low-cost options or healthy foods visible)

5. Disclosure (for example, of the economic or environmental costs associated with energy use, or the full cost of certain credit cards - or large amounts of data, as in the cases of data.gov and the Open Government Partnership, see opengovernmentpartnership.org)

6. Warnings, graphic or otherwise (as for cigarettes)

7. Precommitment strategies (by which people commit to a certain course of action) 
8. Reminders (for example, by e-mail or text message, as for overdue bills and coming obligations or appointments)

9. Eliciting implementation intentions ("Do you plan to vote?")

10. Informing people of the nature and consequences of their own past choices ("smart disclosure" in the United States and the "midata project" in the United Kingdom)

\section{INSTITUTIONALIZING NUDGES: TWO APPROACHES}

What is the best method for implementing nudges? It is certainly possible to rely entirely on existing institutions. We could imagine a system in which an understanding of nudges is used by current officials and institutions, including leaders at the highest levels. For example, the relevant research could be enlisted by those involved in promoting competitiveness, environmental protection, public safety, consumer protection, and economic growth - or in reducing private and public corruption and combating poverty, infectious diseases, and obesity. Focusing on concrete problems rather than abstract theories, officials with well-established positions might be expected to use that research, at least on occasion.

If the relevant officials have both knowledge and genuine authority, they might be able to produce significant reforms, simply because they are not akin to a mere research arm or a think-tank. (Even a single person, if given the appropriate authority and mission, could have a large impact.) On one model, the relevant officials would not engage in new research, or at least not in a great deal of it. They would build on what is already known (and perhaps have formal or informal partnerships with those in the private sector who work on these issues). In an important sense, this approach is the simplest, because it does not require new offices or significant additional funding but only attention to the relevant issues and a focus on the right appointments. In the United States, this kind of approach has proved highly successful, with the adoption of numerous nudges.

A quite different approach would be to create a new institution such as a behavioral-insights team or a "nudge unit" of some sort. Such an institution could be organized in different ways, and it 
could have many different forms and sizes. On a minimalist model, it would have a small group of knowledgeable people (say, five), bringing relevant findings to bear and perhaps engaging in, or spurring, research on their own. On a more ambitious model, the team could be larger (say, thirty or more), engaging in a wide range of relevant research. A behavioral-insights team could be created as a formal part of government (the preferred model, to ensure real impact) or could have a purely advisory role.

Whatever the precise form of this approach, its advantage is that it would involve a dedicated and specialized team, highly informed and specifically devoted to the relevant work, and with expertise in the design of experiments. If the team could work with others to conduct its own research, including randomized controlled trials, it might be able to produce important findings (as has in fact been done in the United Kingdom and the United States, and with similar efforts that are occurring elsewhere). The risk is that such a team would be akin to an academic adjunct, a kind of outsider, without the ability or power to initiate real reform. Authority greatly matters. The United Kingdom has had the most experience with this kind of approach, and it has succeeded in part because it has enjoyed highlevel support and access.

In this domain, one size does not fit all, but it is noteworthy that a growing number of nations have concluded that it is worthwhile to have a dedicated team. Of course, the two approaches might prove complementary.

\section{THE WAY FORWARD}

This book offers an exceedingly valuable exploration of what it means for both private and public organizations to be behaviorally informed. It is full of both findings and ideas. It is based in large part on the inspiring work of the BI.Org partnership, which includes many of the best researchers, working at the frontiers of theory and practice. These include the Behavioural Insights Team from the United Kingdom (now working in dozens of nations), the World Bank, ideas42, and BEAR, along with prominent academic researchers. 
For all the progress, a great deal remains to be done. Nudging, and behaviorally informed approaches in general, are out of their infancy - but still in their early teenage years. There is so much more to be done, to save money, to improve health, to prevent premature death, and to increase well-being in general. The nudgers themselves need to be nudged. This book helps point the way. 
This page intentionally left blank 